\title{
Impact of Serum Progesterone Value on Embryo Transfer Timing, and Pregnancy Rate
}

\author{
Mustafa Zakaria ${ }^{*}$, Noureddine Louanjli' ${ }^{2}$, Aya Al-ibraheemi ${ }^{3}$, Mohamed Ennaji ${ }^{4}$, Wassym R. Senhaji ${ }^{5}$, \\ Abdelhafid Natiq ${ }^{6}$, Romaissa Boutiche ${ }^{7}$, Modou M. Mbaye ${ }^{8}$, Mohamed Zarqaoui ${ }^{9}$
}

${ }^{1}$ MD. Senior Clinical Embryology and Assisted Reproduction, Deputy Executive Director and Administrative Coordinator of the Association for Scientific Research of the IRIFIV-AISRG Group, Consultant in IRIFIV Fertility Center, IVF Laboratoy, Casablanca, Morocco

${ }^{2}$ Head of the LABOMAC Laboratory and IRIFIV Fertility Center, AFC Fertility Center, Casablanca, Morocco

${ }^{3}$ UK. Researcher in the Scientific Research Group and Member of the Association for Scientific Research of the IRIFIV-AISRG Group, Liverpool, England

${ }^{4}$ Member of the Association for Scientific Research of the IRIFIV-AISRG Group, Casablanca, Morocco

${ }^{5}$ GYN OBT, Member of the Association for Scientific Research of the IRIFIV-AISRG Group, Casablanca, Morocco

${ }^{6} \mathrm{PhD}$ in Science in Genetic Specializing in Department of Medical Genetics National Institute of Health Rabat, Morocco

Researcher in the ART IRIFIV Scientific Research Group, Casablanca, Morocco

${ }^{7}$ Laboratory IVF Algeria, Rotary Fertility Center, Member of the Association for Scientific Research of the IRIFIV-AISRG Group,

Algiers, Algeria

${ }^{8}$ Laboratory of Physiopathology, Genetics Molecular and Biotechnology (PGMB), Faculty of Sciences Ain Chock, Research Center, Health and Biotechnology, University Hassan, Casablanca, Morocco

${ }^{9}$ GYN OBT, Head of the Association for Scientific Research of the IRIFIV-AISRG Group, Casablanca, Morocco

Email: ^info@irifiv-aisrg.com, ${ }^{\star}$ dr.zakaria@irifiv-aisrg.com

How to cite this paper: Zakaria, M., Louanjli, N., Al-ibraheemi, A., Ennaji, M., Senhaji, W.R., Natiq, A., Boutiche, R., Mbaye, M.M. and Zarqaoui, M. (2021) Impact of Serum Progesterone Value on Embryo Transfer Timing, and Pregnancy Rate. Open Access Library Journal, 8: e7414.

https://doi.org/10.4236/oalib.1107414

Received: April 13, 2021

Accepted: July 6, 2021

Published: July 9, 2021

Copyright (C) 2021 by author(s) and Open Access Library Inc.

This work is licensed under the Creative Commons Attribution International License (CC BY 4.0).

http://creativecommons.org/licenses/by/4.0/

\begin{abstract}
The impact of progesterone on pregnancy and supporting embryo implantation prior embryo transfer in IVF treatment have been well studied and several studies proved and demonstrated the positive impact of $\mathrm{P} 4$ on pregnancy. However, this impact can be influenced by various factors such as timing of P4 administration and P4 dosage. Moreover, there are different types of P4 administration such as oral and vaginal $\mathrm{P} 4$ supplementation, which can alter the impact of $\mathrm{P} 4$ on pregnancy rate. This literature article discusses the function of progesterone, the effect of P4 on embryo transfer and the optimal level of serum before embryo transfer.
\end{abstract}

\section{Subject Areas}

Gynecology \& Obstetrics

\section{Keywords}

Progesterone Function, Serum Progesterone and Embryo Transfer, Progesterone 
Supplementation Route and Administration Timing, Why Optimal P4 Level Is Vital

\section{Introduction}

Progesterone is one of the steroids hormones, which performs an essential function in many tissues other than the reproductive system, like in the mammary gland, to prepare the glands for breastfeeding, the cardiovascular system, central nervous system and bones. Firstly, progesterone was described as a molecular formula; yet, only in the mid-1940s, Russell Marker begins to manufacture progesterone from diosgenin, derived from the Japanese plant Dioscorea tokoro. Progesterone provides the endometrial transition from a proliferative to the secretary stage, support embryo development and is vital to pregnancy maintenance [1]. The corpus luteum substantially produces progesterone until the ninth week of pregnancy; following, the trophoblasts improve progesterone production, to become the most extensive resource of this hormone after the 12th-week gestation. Corpus luteum lack appears to happen in 35\% of recurrent miscarriage cases. Progesterone is essentially manufactured from maternal LDL cholesterol through the placenta by a total enzyme system, and only a tiny portion will produce by fetal steroid genesis [1]. Progesterone promotes interaction on particular receptors by inducing tubal motility and impacting endometrial maturation and uterine vascularization in the pre-implantation phase. Moreover, $\mathrm{P} 4$ inhibits the $\mathrm{T}$ lymphocyte-mediated tissue reaction, collectively with human chorionic gonadotrophin and decidual cortisol. Progesterone appears to produce tocolytic and immunosuppressive influences in the areas of significant contact within maternal and fetal compartments.

\section{Progesterone Function}

P4 is an intrafollicular steroid that performs an essential role in ovulation, implantation, and pregnancy support and maintenance. P4 is short and elevated at 18 hours following the luteinizing hormone (LH) surge release; in addition, P4 is the main content of follicular fluid steroids in mammalian preovulatory follicles [2] P4 was initially being considered as a contraceptive factor through reducing the luteinizing hormone surge and ovulation. It has an essential function in pregnancy maintenance and controlling biological processes in the ovarian tissue and fetomaternal unit. The processes consist of meiosis resumption, fertilization, embryonic development and implantation [2]. Clinically, it can be utilised in the female reproductive system as luteal support during in vitro fertilisation (IVF), hormone replacement treatment for older women, and as a treatment for endometriosis and polycystic ovarian syndrome in younger women. Moreover, P4 has immunological roles for the maintenance of a fetomaternal. Some researches illustrate that $\mathrm{P} 4$ treatment for luteal maintenance in- 
creased uterine receptivity at the ultrastructure levels and improved mice's implantation rate [2]. The function of steroids is to get meiotic support and undergo regular fertilisation and development to the blastocyst stage. The higher the ratios of P4 to E2 in follicular fluid in several species, the better embryo development. In one research, scientists tried to examine the impact of $\mathrm{P} 4$ on the developmental capability of mouse GV oocytes and following fertilisation potential in concentrations comparable to that of preovulatory follicular fluid. The results pointed that $\mathrm{P} 4$ could not improve the embryo's fertilisation rate and development to the blastocyst stage. Other studies have reported similar results, which indicates that the $\mathrm{P} 4$ maturation medium reduced the rate of blastocyst formation [2]. However, on the other hand, another research showed that the presence of $\mathrm{P} 4$ in the porcine oocyte maturation medium improved sperm head decondensation and male pronuclei formation; moreover, they found out that the addition of $\mathrm{P} 4$ to the porcine oocyte maturation medium can increase both fertilisation and cleavage rates, whereas E2 could not [2]. The importance of sufficient progesterone systemic concentrations through early embryo development is recommended by several researchers, including supplementation with exogenous progesterone. Progesterone's effects can be made both by a direct impact on the embryo or indirectly via the uterus or possibly both. As for the direct impact, progesterone receptors (PGRs) (Figure 1) must be present in the embryo; however, PGRs in mice couldn't be found until the blastocyst stage. Therefore, it is most likely for progesterone to affect embryo survival through an indirect effect on the uterus [3]. The indirect effect of P4 can impact embryo development by binding to uterine stroma or endometrial PGRs and starting a process of events containing differences in gene expression; thus, eventually protein expression or variations in uterine permeability to ions, amino acids or metabolites from plasma or by non-genomic effects on the uterine endometrium [3].

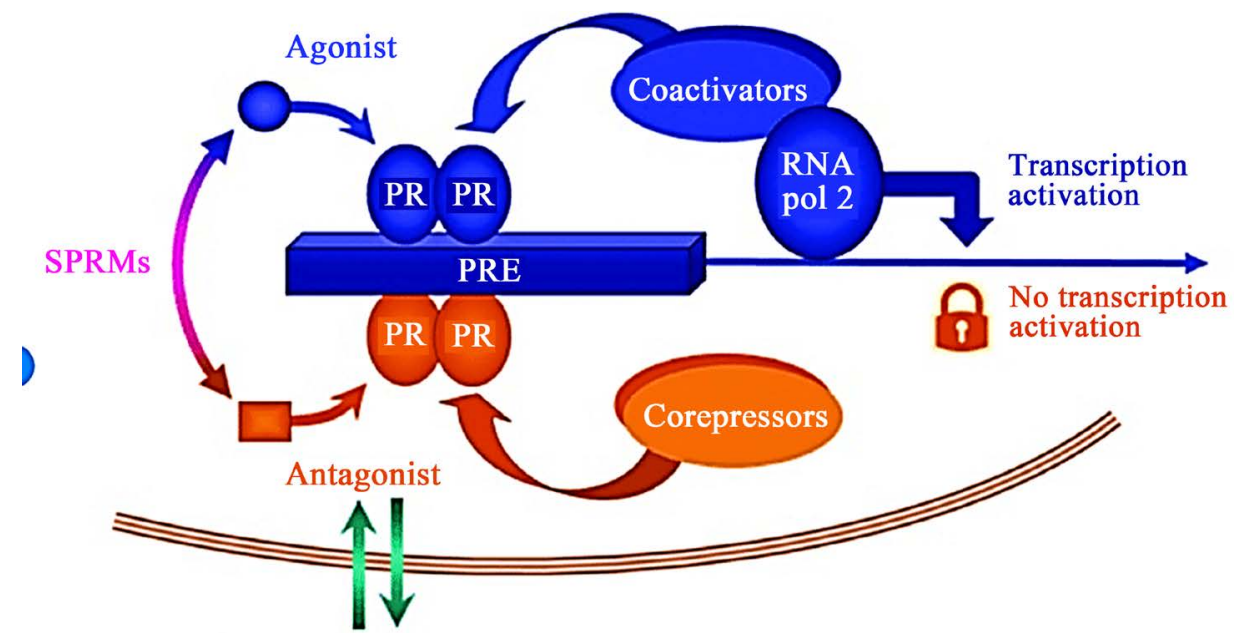

Figure 1. Action mechanism of selective progesterone receptor modulators SPRMs, that have a direct effect on uterine fibroids, endometrium and the pituitary gland; where it binds to the PR to medicate action either by activation or deactivation [3]. 


\section{Serum Progesterone and Embryo Transfer}

Several early studies suggested the importance of luteal phase support in frozen cycles, which has shown that progesterone's supplementation does impact the outcome in frozen embryo transfer. Progesterone (P4) is needed for successful embryonic implantation into the endometrium and support of the pregnancy in natural cycles, fresh in vitro fertilization cycles, and frozen embryo transfer (FET) cycles. Thus, rumours investigation has been done to illustrates the impact and importance of the P4 in Embry transfer. Therefore, P4 must be supplemented in order to enhance embryo transfer success and pregnancy rate.

\subsection{Progesterone Supplementation Route and Administration Timing}

For $\mathrm{P} 4$ supplementation, there are three administration routes, either oral, vaginal or intramuscular. The oral $\mathrm{P} 4$ administration provides inadequately sustained plasma progesterone concentrations, although plasma progesterone concentration is a weak marker of bio-availability [4]. Regarding vaginal and intramuscular administration, it has been reported that intramuscular administration is more effective than vaginal supplementation [5]. Several factors can change P4 serum levels following vaginal administration, like sexual intercourse, poor patient appliance, vaginal retention, disposition, and metabolism variations. Low P4 levels were recorded in more than one-third of patients who had daily vaginal P4 administration of $600 \mathrm{mg}$ micronized progesterone. However, a high vaginal $P$ dose of $1200 \mathrm{mg}$ can increase the serum P levels [6]. P4 route of administration effectiveness can also depends on the other factors, such as age and weight. For instance, in older women, the vaginal progesterone is more efficient as the vaginal mucosa of older women is thinner and more atrophic, increasing the absorption of vaginal progesterone. Moreover, other studies showed that body weight is an independent factor influencing serum progesterone concentrations following four days of vaginal progesterone administration. These results are biologically probable since bodyweight is a vital factor that affects drug absorption, distribution, metabolism and elimination [7]. P4 can be supplemented through Gonadotropin-releasing hormone (GnRH) agonists. GnRH agonists enhance GnRH production, which stimulates stimulating follicle hormone (FSH) and luteinizing hormone (LH), which excites ovulation and develops corpus luteum, that in return stimulates $\mathrm{P} 4$ production (Figure 2) [8].

Another important aspect to consider in $\mathrm{P} 4$ administration is the timing and duration of the administration. The implantation window is the ideal length of progesterone supplementation and the growth of maximal endometrial receptivity which can be determined through endometrial biopsies collection and assessing presence of pinopodesorby other biomarkers of implantation (e.g., the expression of @Vb3, PP14, and HOXA 10 gene expression); also it can be determined through transferring the embryos and examine the pregnancy and implantation rates. For most ART treatment, progesterone supplement practice is 


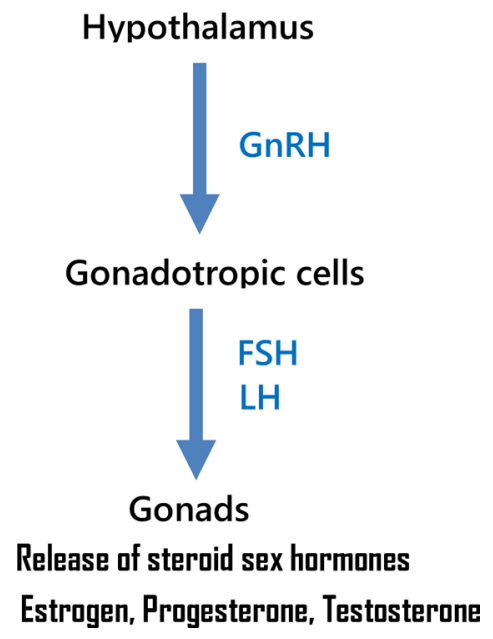

Figure 2. GnRH action mechanism. P4 is produce through a signaling cascade which starts from the hypothalamus in the brain till it reaches the gonads to produce $\mathrm{P} 4$ [8].

three days before embryo transfer; thus, if an embryo transfer is performed on Day 3 of development, progesterone supplement should be performed three days before embryo 3. Therefore, progesterone exposure days and timing before embryo transfer relay on the frozen embryos' stage to be transferred. In Sharma and Majumdar, progesterone supplementation for three days before embryo transfer (Embry at cleavage stage 4 - 6-cell stage) had significantly higher pregnancy and implantation rates than four days of progesterone administration. Therefore, they concluded that progesterone supplementation for three days before embryo transfer had better pregnancy and implantation rates (Figure 3) [9].

\subsection{Progesterone Level and Embryo Transfer}

$\mathrm{P}$ has a fundamental function in the endometrial transformation before frozen embryo transfer (FET). Endometrial transformation can be accomplished through natural cycle or via an artificial endometrial preparation [10]. Natural endometrial transformation requires no external medication, where corpus luteum secrete endogenous progesterone in an ovulatory cycle and, thus making this approach preferable to some patients. In artificial endometrial transformation, external administration of exogenous estrogen and progesterone is applied to accomplish both adequate endometrial priming and serum hormonal values approaching the natural ovulatory cycle [10]. Progesterone serum level is vital to achieve successful implementation and pregnancy rate on the day of embryo transfer, several studies investigate abnormal P4 serum level (low and elevated level), and their impact on embryo transfer, pregnancy rate, and live birth. In one study, a significant association was reported among positive pregnancy outcome and progesterone level at the day of hCG trigger were $79.2 \%$ of a female with positive pregnancy at progesterone level $<1.5$, while $9.3 \%$ of a female with positive pregnancy at progesterone level $>2.5$. In addition, 1107 women get pregnant when level of progesterone $<1.5 \mathrm{ng} / \mathrm{mL}, 127$ get pregnant at level of $1.5-2 \mathrm{ng} /$ $\mathrm{ml}, 70$ get pregnant at level of $>2-2.5 \mathrm{ng} / \mathrm{ml}$ (Table 1) [11]. 
(a)

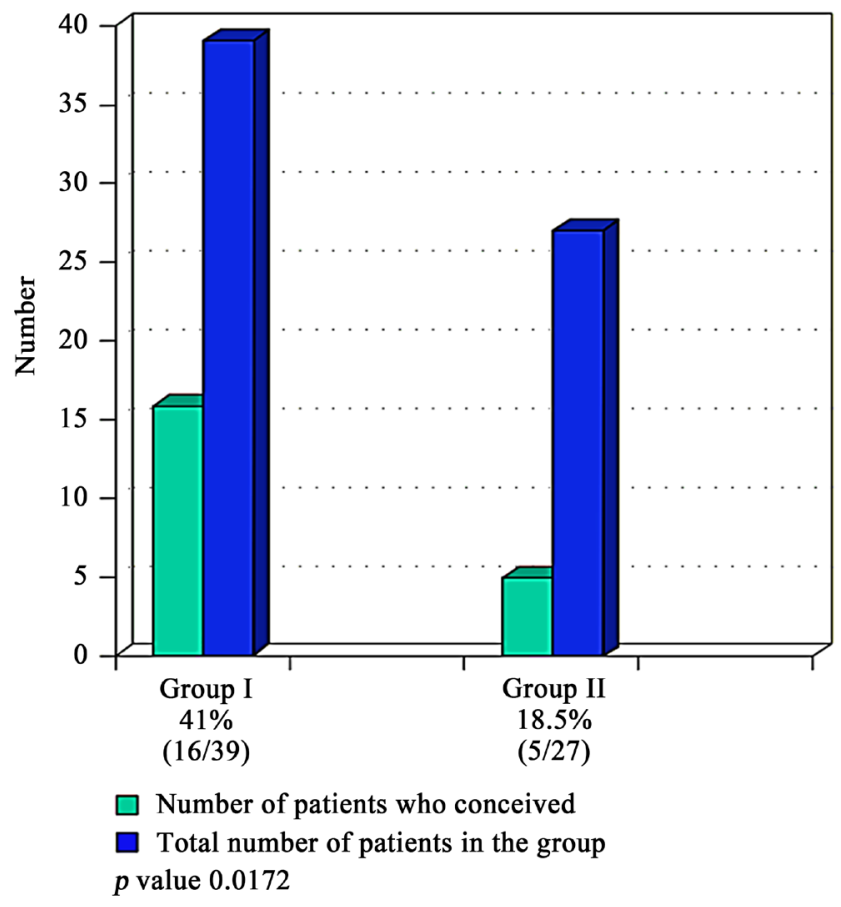

(b)

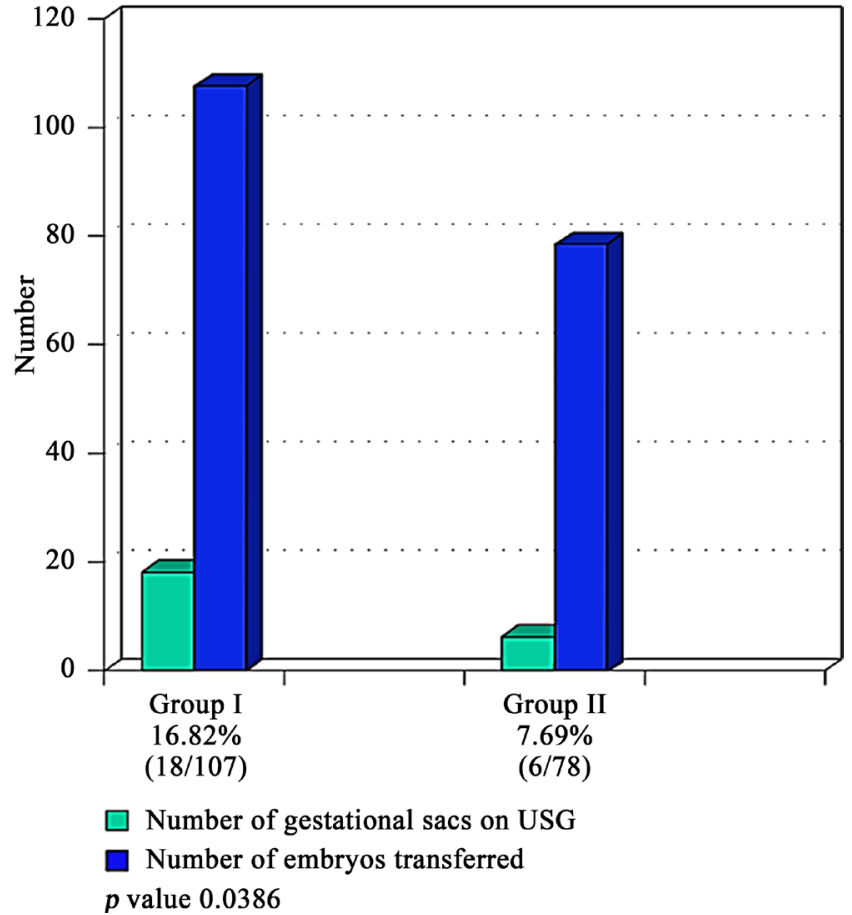

Figure 3. (a) Pregnancy percentage in patients receiving progesterone for 3 and 4 days before embryo transfer. (b) Implantation rates in patients receiving progesterone for 3 and 4 days before embryo transfer [9].

Table 1. Relationship among progesterone level at day of HCG trigger and pregnancy outcome (age 20 - 45) [11].

\begin{tabular}{ccc}
\hline \multirow{2}{*}{ Study variables } & \multicolumn{2}{c}{ pregnancy } \\
\cline { 2 - 3 } & Positive & Negative \\
\hline Less than 0.9 & $358(49.7)$ & $353(47.9)$ \\
0.9 -below & $210(29.2)$ & $184(25.0)$ \\
$1.5-2$ & $56(7.8)$ & $75(10.1)$ \\
$2-2.5$ & $31(4.3)$ & $39(5.3)$ \\
More than 2.5 & $65(9.0)$ & $86(11.7)$ \\
Total & $720(100.0)$ & $736(100.0)$ \\
\hline
\end{tabular}

Moreover, Gaggiotti-Marre et al. study showed the importance of serum levels on the day prior to FET in women undergoing a natural endometrial preparation cycle; their results indicated that low serum $\mathrm{P}$ levels on the day before embryo transfer $(<10 \mathrm{ng} / \mathrm{ml})$ are correlated with significantly lower clinical pregnancy rate and live birth rate. Also, low serum P4 level was associated with higher miscarriage rates were (Figure 4) [11].

Similar results to the above studies have been reported, which showed that patients with an artificial endometrial preparation cycle using vaginal progesterone had a significantly reduced ongoing pregnancy rate, as the serum, $\mathrm{P}$ was $<9.2$ 


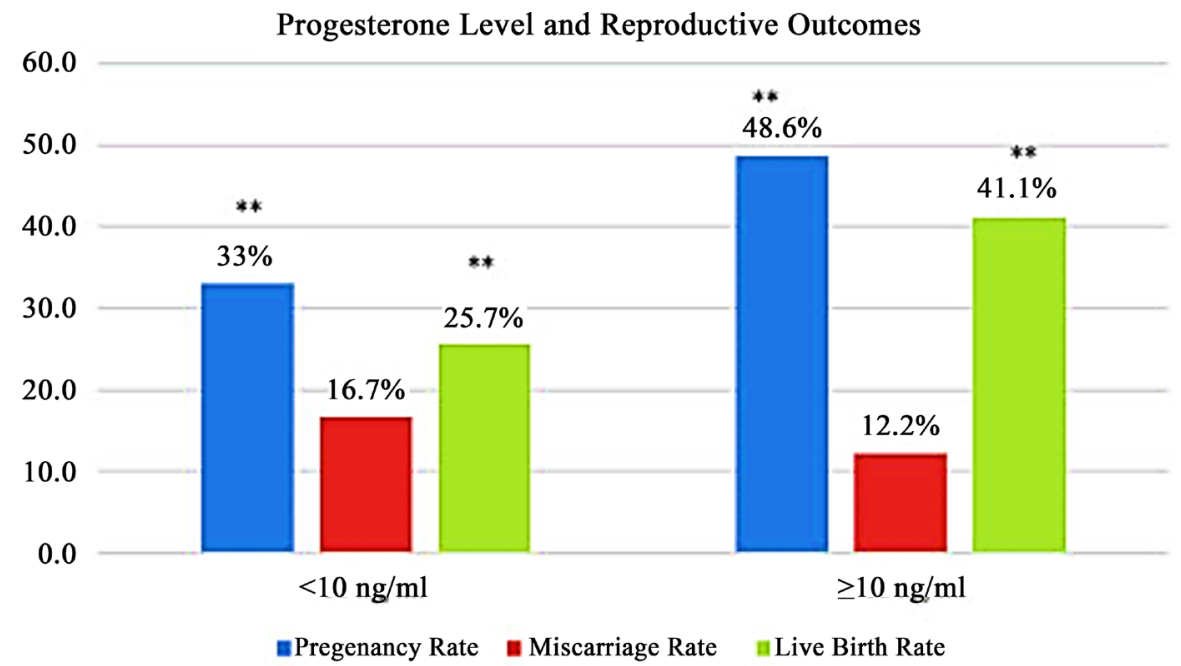

Figure 4. Progesterone level and embryo transfer outcome. The results indicate that P4 level $>10 \mathrm{ng} / \mathrm{ml}$, is associated with better reproductive outcomes [11]. $\left.{ }^{\star}\right)$ Indicate "p" values: ${ }^{* *}$ highly significant $(\mathrm{p}<0.01)$.

$\mathrm{ng} / \mathrm{ml}$ on the day of embryo transfer [12]. Moreover, Labarta et al., observation point out no association for endometrial volume with either serum $\mathrm{P}$ levels or ongoing pregnancy rate, which implies if a minimal endometrial thickness is obtained, serum P levels would be the primary, more predictable marker for pregnancy more than ultrasound endometrium evaluations. Therefore, a minimal serum $\mathrm{P}$ threshold on the day of embryo transfer should be reached for a successful ongoing pregnancy rate [12]. The retrospective performed by SantosRibeiro et al., 2014, also evaluates the effect of low progesterone on the live-birth rate. The study conducted several evaluations according to various ordinal and normal progesterone levels $(\leq 0.50,0.50-0.75,0.75-1.00,1.00-1.25,1.25-$ $1.50,>1.50 \mathrm{ng} / \mathrm{ml}$ )" to compare the difference in live birth percentage. According to the previous serum progesterone levels, the live birth rates were $17.1 \%, 25.1 \%$, $26.7 \%, 25.5 \%, 21.9 \%$ and $16.6 \%$. The results confirm earlier studies conclusion by finding the association between low $\mathrm{P} 4$ level and low birth rate [13]. Moreover, all patients had the same dose of exogenous FSH similar to the regular $\mathrm{P}$ group; they were also simulated using the same hCG criteria and had unaffected maturation and fertilization rates. Hence, the low birth weight associated with a low P4 level does not appear to be related to inadequate stimulation or poorer oocyte maturation or fertilization competence [13]. Throughout controlled ovarian hyperstimulation $(\mathrm{COH})$, progesterone serum elevation is always avoided through suppressing luteinizing hormone (LH) secretion by administrating a gonadotropin releasing hormone $(\mathrm{GnRH})$ agonist or antagonist. Therefore, various researches have been performed to record and evaluate elevated P4 impact on embryo transfer and pregnancy rate. A research examined the incidence of premature progesterone elevations in frozen-thawed embryo transfer cycles and the association with any adverse impacts on clinical outcomes. The elevated serum progesterone level was $4.6 \mathrm{nmol} / \mathrm{l}$ or higher in over one-fifth of patients 
undergoing IVF treatment. The results indicated no difference in clinical pregnancy rate, and ongoing pregnancy rate was seen within patients with and without elevated progesterone [14]. However, it has been demonstrated that High P level prior to oocyte collection is linked with a noticeable endometrial receptivity reduction. Also, definitely the gene expression profile of the endometrium will be affected when $\mathrm{P}$ level is higher than its normal level at the end of the follicular phase. Elevated P levels on the day of hCG throughout the first fresh cycle are associated with poor pregnancy rate, although not in following frozen-thawed embryo transfer cycles [15]. This finding has been supported by Ibrahim et al., where they demonstrate that the impact of elevated P4 can be extended to the level of fetal development itself and results in lower birth weights. The study also recommends that the progesterone on the day of hCG administration may be reasonable to delay the transfer in such circumstances. The research also points out that high progesterone on the day of hCG administration can be lined with various vascular endothelial growth factor expression in the endometrium. Accordingly, this can alter endometrial receptivity, ending in reduced implantation. Thus, it has been reported that that elevated progesterone can be correlated with adverse perinatal results [16]. Moreover, in one of the respective researches, they assessed whether the hormone administration following the end of menstruation and till the day of hCG administration in GnRH antagonist/recombinant FSH cycles would be associated with pregnancy rate. The results suggested that elevated P4 serum level through the menstruation-free interval is correlated with a reduced likelihood of ongoing pregnancy [17]. The impaired influence of high $\mathrm{P}$ exposure on pregnancy rate can be assigned to the increase and premature expression of $\mathrm{P} 4$ receptors on the endometrium in stimulated IVF cycles, which has been linked with the elevated E2 concentration through the follicular phase in these cycles [17]. Furthermore, in the Bosch et al., study where they also evaluate elevated progesterone, they demonstrated no inverted association between ongoing pregnancy rates and serum progesterone levels on the day of hCG administration. In patients with elevated progesterone serum levels, $<$ or $=1.5 \mathrm{ng} /$ $\mathrm{mL}$, significantly higher ongoing pregnancy rates were recorded in comparison with patients with normal serum progesterone levels $>1.5 \mathrm{ng} / \mathrm{mL}$ (31.0\% vs $19.1 \%$; $\mathrm{P}=0.00006$ ); the odds ratio was 0.53 , with a $95 \%$ confidence interval of 0.38 to 0.72 [18]. The study also showed that elevated progesterone could be caused by an increase in a daily dosage of follicle-stimulating hormone, the number of oocytes collected, and estradiol (E2) values on the day of hCG administration. Additionally, women treated with GnRH agonists had significantly higher serum progesterone levels than those receiving GnRH antagonists ( $0.84 \pm 0.67$ vs $0.75 \pm$ $0.66 \mathrm{ng} / \mathrm{mL} ; \mathrm{P}=0.0003$ ) [18]. Finally, Ochsenkühn et al., also confirmed the above researches finding by proposing that high follicular $\mathrm{P}$ promotes the endometrium, and hence the replacement of a day- 3 embryo in an asynchronous endometrium point out a failure of establishing embryo-endometrium cross-dialogue, this leading to embryo death and implementation failure. The study pro- 
posed that women who were experiencing pituitary down-regulation in addition to $\mathrm{COH}$ with by $\mathrm{GnRH}$ agonists and 5 days following collection display a significantly reduced live birth rate in the case of a slight P4 rise on the day of hCG administration [1].

\subsection{Why Optimal P4 Level Is Vital}

Low serum progesterone can be a factor of low pregnancy rate; thus, this can be more serious with patients who already have low P4 in their blood circulation. According to González-Foruria et al., study, it has been found that certain factors affect progesterone concentrations on the day before embryo transfer, which can alter pharmacokinetics such as age, weight, and prior history of low progesterone concentrations, however other factors such as the timing of blood sampling do not depend on differences in drug absorption [7]. In addition, high P4 serum level has been High P level before eggs collection is linked with a noticeable endometrial receptivity decrease. The endometrium's gene expression profile at the end of the follicular phase is certainly altered when the P level is above 1.5 $\mathrm{ng} / \mathrm{ml}$ [15]. Progesterone importance in successful pregnancy rate and implementation rate have been clearly illustrated, however, significant progesterone elevation prior to hCG injection can point to a desynchronization within the endometrium and the embryo through reducing pregnancy rates [14]. In summary, a successful implantation needs a competent blastocyst synchronization with a receptive endometrium, which is mainly coordinated by the endometrium receptivity are estrogen and progesterone. Thus, an optimal P4 level is essential for successful implantation and pregnancy [15].

\section{Conclusion}

Progesterone is essential for establishing and maintaining embryo implantation and pregnancy; also, P4 is used for luteal phase deficiency in infertility treatment. Yet, progesterone therapy's optimal timing and dose can influence the impact of P4 on pregnancy. Also, low or elevated P4 level can negatively impact embryo transfer timing and pregnancy rate.

\section{Acknowledgements}

Objective of the Association for Scientific Research of the IRIFIV-AISRG Group (IRIFIV-AISRG),Research foundation in Casablanca, Maintaining consistent and reliably high success rates is a monthly challenge for in IVF labs, the IRIFIV Fertility Center in Casablanca-Morocco Department of Reproductive Medicine and Reproductive Biology and Embryology, advocacy of interdisciplinary Department of Reproductive Medicine and Reproductive Biology and Embryology study, encompassing the areas of research, collections and publishing Articles.

\section{Conflicts of Interest}

The authors declare no conflicts of interest. 


\section{References}

[1] Taraborrelli, S. (2015) Physiology, Production and Action of Progesterone. Acta Obstetricia et Gynecologica Scandinavica, 94, 8-16. https://doi.org/10.1111/aogs.12771

[2] Salehnia, M. and Zavareh, S. (2013) The Effects of Progesterone on Oocyte Maturation and Embryo Development. International Journal of Fertility \& Sterility, 7, 7481.

[3] Morris, D. and Diskin, M. (2008) Effect of Progesterone on Embryo Survival. Animal, 2, 1112-1119. https://doi.org/10.1017/S1751731108002474

[4] Penzias, A. (2002) Luteal Phase Support. Fertility and Sterility, 77, 318-323. https://doi.org/10.1016/S0015-0282(01)02961-2

[5] Jobanputra, K., Toner, J., Denoncourt, R. and Gibbons, W. (1999) Crinone 8\% (90 $\mathrm{mg}) *$ Given Once Daily for Progesterone Replacement Therapy in Donor Egg Cycles 2. Fertility and Sterility, 72, 980-984. https://doi.org/10.1016/S0015-0282(99)00390-8

[6] Urman, B., Alatas, C., Aksoy, S., Mercan, R., Isiklar, A. and Balaban, B. (1999) Elevated Serum Progesterone Level on the Day of Human Chorionic Gonadotropin Administration Does Not Adversely Affect Implantation Rates after Intracytoplasmic Sperm Injection and Embryo Transfer. Fertility and Sterility, 72, 975-979. https://doi.org/10.1016/S0015-0282(99)00421-5

[7] González-Foruria, I., Gaggiotti-Marre, S., Álvarez, M., Martínez, F., García, S., Rodríguez, I., Coroleu, B. and Polyzos, N. (2021) Factors Associated with Serum Progesterone Concentrations the Day before Cryopreserved Embryo Transfer in Artificial Cycles. Reproductive BioMedicine Online, 40, 797-804.

https://doi.org/10.1016/j.rbmo.2020.03.001

[8] Bustillo, M., Stern, J.J. and Coulam, C.B. (1995) Serum Progesterone at the Time of Human Chorionic Gonadotrophin Does Not Predict Pregnancy in In-Vitro Fertilization and Embryo Transfer. Human Reproduction, 10, 2862-2867.

https://doi.org/10.1093/oxfordjournals.humrep.a135808

[9] Sharma, S. and Majumdar, A. (2016) Determining the Optimal Duration of Progesterone Supplementation prior to Transfer of Cryopreserved Embryos and Its Impact on Implantation and Pregnancy Rates: A Pilot Study. International Journal of Reproductive Medicine, 2016, Article ID: 7128485. https://doi.org/10.1155/2016/7128485

[10] Gaggiotti-Marre, S., Álvarez, M., González-Foruria, I., Parriego, M., Garcia, S., Martínez, F., Barri, P., Polyzos, N. and Coroleu, B. (2020) Low Progesterone Levels on the Day before Natural Cycle Frozen Embryo Transfer Are Negatively Associated with Live Birth Rates. Human Reproduction, 35, 1623-1629.

https://doi.org/10.1093/humrep/deaa092

[11] Albdairi, A. and Hussein, H. (2020) The Level of Serum Progesterone on the Day of HCG Administration in Assisted Reproduction Cycles. Medico-Legal Update, 20, 677-682.

[12] Labarta, E., Mariani, G., Holtmann, N., Celada, P., Remohí, J. and Bosch, E. (2017) Low Serum Progesterone on the Day of Embryo Transfer Is Associated with a Diminished Ongoing Pregnancy Rate in Oocyte Donation Cycles after Artificial Endometrial Preparation: A Prospective Study. Human Reproduction, 32, 2437-2442. https://doi.org/10.1093/humrep/dex316

[13] Santos-Ribeiro, S., Polyzos, N., Haentjens, P., Smitz, J., Camus, M., Tournaye, H. 
and Blockeel, C. (2014) Live Birth Rates after IVF Are Reduced by Both Low and High Progesterone Levels on the Day of Human Chorionic Gonadotrophin Administration. Human Reproduction, 29, 1698-1705.

https://doi.org/10.1093/humrep/deu151

[14] Groenewoud, E., Macklon, N., Cohlen, B., Al-Oraiby, A., Brinkhuis, E., Broekmans, F., de Bruin, J., van der Dool, G., Fleisher, K., Friederich, J., Goddijn, M., Hoek, A., Hoozemans, D., Kaaijk, E., Koks, C., Laven, J., van der Linden, P., Manger, A., van Rumpste, M. and Spinder, T. (2017) The Effect of Elevated Progesterone Levels before HCG Triggering in Modified Natural Cycle Frozen-Thawed Embryo Transfer Cycles. Reproductive BioMedicine Online, 34, 546-554. https://doi.org/10.1016/j.rbmo.2017.02.008

[15] Liang, Y., Liu, L., Jin, Z., Liang, X., Fu, Y., Gu, X. and Yang, Z. (2018) The High Concentration of Progesterone Is Harmful for Endometrial Receptivity and Decidualization. Scientific Reports, 8, Article No. 712.

https://doi.org/10.1038/s41598-017-18643-w

[16] Ibrahim, Y., Haviland, M., Hacker, M., Penzias, A., Thornton, K. and Sakkas, D. (2017) Elevated Progesterone and Its Impact on Birth Weight after Fresh Embryo Transfers. Journal of Assisted Reproduction and Genetics, 34, 759-764. https://doi.org/10.1007/s10815-017-0920-8

[17] Kyrou, D., Kolibianakis, E., Fatemi, H., Camus, M., Tournaye, H., Tarlatzis, B. and Devroey, P. (2011) High Exposure to Progesterone between the End of Menstruation and the Day of Triggering Final Oocyte Maturation Is Associated with a Decreased Probability of Pregnancy in Patients Treated by in Vitro Fertilization and Intracytoplasmic Sperm Injection. Fertility and Sterility, 96, 884-888. https://doi.org/10.1016/j.fertnstert.2011.07.1101

[18] Bosch, E., Labarta, E., Crespo, J., Simón, C., Remohí, J., Jenkins, J. and Pellicer, A. (2011) Circulating Progesterone Levels and Ongoing Pregnancy Rates in Controlled Ovarian Stimulation Cycles for in Vitro Fertilization: Analysis of over 4000 Cycles. Obstetrical \& Gynecological Survey, 66, 27-28.

https://doi.org/10.1097/OGX.0b013e31820220dd

\author{
Abbreviations \\ In-vitro fertilization (IVF) \\ luteinizing hormone ( $\mathrm{LH})$ \\ progesterone receptors (PGRs) \\ selective progesterone receptor modulators (SPRMs) \\ frozen embryo transfer (FET) \\ Gonadotropin-releasing hormone ( $\mathrm{GnRH}$ ) \\ follicle hormone (FSH) \\ estradiol (E2)
}

